\title{
Decoherence of a Superposition of Macroscopic Current States in a SQUID
}

\author{
E. M. Chudnovsky ${ }^{1}$ and A. B. Kuklov ${ }^{2}$ \\ ${ }^{1}$ Department of Physics and Astronomy, Lehman College, City University of New York, \\ 250 Bedford Park Boulevard West, Bronx, New York 10468-1589 \\ 2 Department of Engineering Science and Physics, The College of Staten Island, \\ City University of New York, Staten Island, New York 10314
}

\begin{abstract}
We show that fundamental conservation laws mandate parameter-free mechanisms of decoherence of quantum oscillations of the superconducting current between opposite directions in a SQUID emission of phonons and photons at the oscillation frequency. The corresponding rates are computed and compared with experimental findings. The decohering effects of external mechanical and magnetic noise are investigated.

PACS numbers: 03.65.Yz, 74.50.+r, 85.25.Dq
\end{abstract}

\section{INTRODUCTION}

The possibility of macroscopic quantum tunneling of the magnetic flux in SQUIDs was suggested in a seminal paper of Caldeira and Leggett 1 and was subsequently demonstrated in experiment 2 . 1 . Following these developments the possibility of coherent quantum oscillations between macroscopic flux states was intensively studied by theorists (see, e.g. Ref. 4 and references therein). Modern interest to this problem was generated by the hope to build a superconducting (SC) qubit. The goal of preparing quantum superposition of macroscopic flux states in a SQUID had remained elusive however, until recent experiments of Friedman et al. 5 and van der Wal et al.6. In these experiments the tunneling splitting between the states corresponding to symmetric and antisymmetric quantum superpositions of clockwise and counterclockwise currents in a SQUID loop have been observed. These experiments were followed by similar measurements of more elaborate designst

The most important question about the above experiments is the one of the decoherence time 6 . Any degree of freedom that interacts with the coherently oscillating variable can be the source of decoherence. One can divide all such sources into two groups, avoidable and unavoidable. Examples of avoidable sources are, e.g., nuclear spins and non-thermal noise. In this paper we study generic mechanisms of decpherence which are controlled by the conservation laws10.11. In application to SQUIDs, such mechanisms are unavoidable as they originate from the fundamental symmetries of free space. To illustrate our point, consider, e.g., experiment of Ref. 5 in which the current, $J \sim 3 \mu \mathrm{A}$, oscillated due to quantum tunneling between clockwise and counterclockwise directions. The angular momentum associated with the current was of order $L_{c} \sim 10^{10} \hbar$. The total angular momentum of the system is $L_{t o t}=L_{c}+L_{m}$, where $L_{m}$ is the mechanical angular momentum of the solid matrix bearing the current. To conserve $L_{t o t}$, oscillations of $L_{c}$ between $L_{c}=10^{10} \hbar$ and $L_{c}=-10^{10} \hbar$ must be accompa- nied by simultaneous torsional oscillations of the solid matrix between the states with the angular momenta $L_{m}=-10^{10} \hbar$ and $L_{m}=10^{10} \hbar$, so that $L_{t o t}=0$. One can argue that the current loop is always firmly attached to a substrate which is firmly attached to another solid, etc., so that the angular momentum is transferred to the infinite mass, like the linear momentum in Mössbauer experiment. If this were true, the SC current would simply bounce elastically between clockwise and counterclockwise directions, making conservation of the total angular momentum irrelevant. It is easy to see, however, that for a current oscillating at a high frequency, e.g., $f_{0} \sim 10^{9}-10^{10} \mathrm{~s}^{-1}$, the analogy with the Mössbauer effect breaks down. Indeed, during the period of oscillations, the elastic deformation cannot travel more than a distance $v_{s} / f_{0}$ away from the current; $v_{s}$ being the speed of the transverse sound. For, e.g., $v_{s} \sim 5 \times 10^{3} \mathrm{~m} / \mathrm{s}$ and $f_{0} \sim 5 \times 10^{9} \mathrm{~s}^{-1}$ one obtains $v_{s} / f_{0} \sim 1 \mu \mathrm{m}$. Thus, the part of the solid involved in the conservation of the angular momentum is small, not macroscopically large. Consequently, the torsional oscillations of the part of the solid matrix "co-wiggling" with the current must generate phonons of frequency $f_{0}$ in the surrounding matter. As long as the speed of sound is finite this should result in a decoherence of quantum oscillations of the current. Similar effect exists due to the interaction of the magnetic moment of the current with vacuum photons. The difference from the phonon problem is that at $f_{0} \sim 5 \times 10^{9} \mathrm{~s}^{-1}$ the wavelength of the light, $\lambda_{l}=c / f_{0}$, is about five orders of magnitude larger than $\lambda_{s}$. Consequently, the vacuum properties of the photons depend strongly on the geometry of the experiment, in particular, on the metal shielding of the SQUID.

The above picture is quasiclassical. From the quantum mechanical point of view, the states of the tunneling SC current are not classified by specific values of the angular momentum. The interaction of the current with the solid matrix entangles the angular momentum states of the current with the angular momentum states of the matrix. In the absence of the external noise, the total an- 
gular momentum is, of course, a well-defined conserved quantum number. In this respect, it is important to understand why the external noise acting on the macroscopic solid matrix (like, e.g., vibration of the building) does not instantaneously destroy the quantum entanglement between the angular momentum states of the current and the angular momentum states of the matrix. We will discuss this issue in the context of the local versus global mechanisms of decoherence.

The paper has the following structure. The decoherence due to the exchange of the angular momentum with the solid matrix is studied in Section II. The limits in which the dimensions of the SQUID loop are smaller and greater than the wavelength of the emitted phonons, $\lambda_{s}=v_{s} / f_{0}$, are considered in the Subsections II-A and II-B correspondingly. The role of the external mechanical noise is discussed in the Subsection II-C. The photon effects are studied in Section III. The decoherence in the unshielded and shielded SQUID are computed in Subsections III-A and III-B, respectively. Conclusions that may be useful for building SQUID-based qubits are summarized in Section IV.

\section{DECOHERENCE DUE TO PHONONS}

Consider quantum oscillations of the current $J$ in a flat loop of spacial dimension $R$ at a frequency $f_{0}$. The borderline between the cases of small and large SQUID studied in this Section is the dimension $R \sim \lambda_{s}$. For $f_{0}$ of the order of a few $\mathrm{GHz}$, it is $R \sim 1 \mu \mathrm{m}$. Obviously, both cases, $R<\lambda_{s}$ and $R>\lambda_{s}$ must be of interest for the ongoing experiments on quantum superposition of SQUID states and in connection with the goal of building a SC qubit.

Let the magnitude of the dimensionless angular momentum associated with the current be $L$. The magnetic moment of the current is

$$
M=\mu_{B} L=J a / c,
$$

where $\mu_{B}$ is the Bohr magneton and $a$ is the area of the loop. Thus, for a given current and given dimensions of the SQUID the value of the oscillating angular momentum of the SC electrons can be found from $L=J a / \mu_{B} c$. For, e.g., a $0.1 \mu \mathrm{m}$ (small) SQUID carrying an electric current of $J \sim 0.1 \mu \mathrm{A}$, this gives $L \sim 10^{2}$, while for a $100 \mu \mathrm{m}$ (large) SQUID carrying an electric current of $J \sim 10 \mu \mathrm{A}$, one obtains $L \sim 10^{10}$.

The dynamical torsional deformations of the lattice are described by the transversal displacement field $\mathbf{u}(\mathbf{r}, t)$ satisfying

$$
\nabla \cdot \mathbf{u}=0 .
$$

These deformations do not affect the density of the ionic lattice and, in the long-wave limit, do not modify electronic states. The minimal, allowed by symmetry, coupling of such deformations with the electric current $\mathbf{j}(\mathbf{r}, t)$ must be proportional to $\int d^{3} r \mathbf{j} \cdot \dot{\mathbf{u}}$. Let us show that the coefficient of proportionality is $\mathrm{m}_{e} / \mathrm{e}$, where $\mathrm{m}_{e}$ and e are bare electron mass and charge. Without loss of generality, one can employ the following argument in order to derive the interaction term. Consider the classical density of the kinetic energy of electrons and ions,

$$
\mathrm{KE}=n_{e} \frac{\mathrm{m}_{e} \mathbf{v}_{e}^{2}}{2}+n_{i} \frac{M_{i} \dot{\mathbf{u}}^{2}}{2},
$$

where $n_{e, i}$ are concentrations of electrons and ions, $M_{i}$ is the ionic mass, and $\mathbf{v}_{e}$ is the velocity field of electrons in the laboratory coordinate frame. Notice now that the electronic current is formed by the electron band states in the coordinate frame co-moving with the lattice. Consequently,

$$
\mathbf{j}=\mathrm{e} n_{e}\left(\mathbf{v}_{e}-\dot{\mathbf{u}}\right) .
$$

Expressing Eq. (3) in terms of physical variables $\mathbf{j}$ and $\mathbf{u}$, one ends up with the coupling of the form $\left(\mathrm{m}_{e} / \mathrm{e}\right)(\mathbf{j} \cdot \dot{\mathbf{u}})$. The generalization of this argument for quantum operators is trivial. It gives the following Hamiltonian of the effective interaction in the laboratory frame:

$$
\mathcal{H}_{\text {int }}=\frac{\mathrm{m}_{e}}{\mathrm{e}} \int d^{3} r \mathbf{j} \cdot \dot{\mathbf{u}} .
$$

This formula is a consequence of the translational symmetry and is, therefore, parameter-free. It can be used as long as $\mathcal{H}_{\text {int }}$ results in a small perturbation of oneelectron energy band.

\section{A. Small SQUID}

We shall start with the case of a small current loop of $R<\lambda_{s}$, in the $X Y$ plane. The shape of the loop will be irrelevant. In the context of its magnetoelastic interaction with the solid matrix it is equivalent to a magnetic atom of angular momentum $L$, imbedded in a solid. We shall denote the states with $\mathbf{L}$ up and $\mathbf{L}$ down by $|\uparrow\rangle$ and $|\downarrow\rangle$ respectively. They are the eigenvalues of $L_{z}$ :

$$
L_{z}|\uparrow\rangle=L|\uparrow\rangle, \quad L_{z}|\downarrow\rangle=-L|\downarrow\rangle .
$$

With no regard for the conservation of the angular momentum, the ground state of the system can be approximated by

$$
|0\rangle=\frac{1}{\sqrt{2}}(|\uparrow\rangle+|\downarrow\rangle),
$$

while the first excited state, with the same accuracy, is

$$
|1\rangle=\frac{1}{\sqrt{2}}(|\uparrow\rangle-|\downarrow\rangle) \text {. }
$$

Let the energy separation of these two states be $\Delta=$ $\hbar \omega_{0}=h f_{0}$. If the energy scale of the experiment does 
not significantly exceed $\Delta$, then any quantum state of the SQUID is a superposition $|\psi\rangle=C_{1}|0\rangle+C_{2}|1\rangle$. One of the mechanisms of decoherence of this superposition, that exists down to zero temperature, is the decay of $|1\rangle$ onto $|0\rangle$ accompanied by the radiation of the quantum of energy $\Delta$.

As has been discussed in the Introduction, the conservation law requires that the oscillations of the current are accompanied by the torsional oscillations of the solid matrix, so that the total angular momentum stays constant (e.g., zero). Such a local wiggling of the matrix must result in the finite probability of the emission of a transverse phonon of frequency $f_{0}$. Since the wavelength of the phonon, $\lambda_{s}$, is large compared to the dimensions of the SQUID, its effect on the SQUID is equivalent to the uniform local rotation of the solid matrix at the position of the SQUID 11. In terms of the deformation field $\mathbf{u}(\mathbf{r}, t)$, the angular velocity of this rotation is given by

$$
\boldsymbol{\Omega}=\frac{1}{2} \nabla \times \dot{\mathbf{u}}
$$

Accordingly, the lattice velocity field at the position of the SQUID is $\dot{\mathbf{u}}=\boldsymbol{\Omega} \times \mathbf{r}$. Substituting this into Eq.([), one finds

$$
\mathcal{H}_{e f f}=\hbar \mathbf{L} \cdot \mathbf{\Omega}
$$

where

$$
\hbar \mathbf{L} \equiv \frac{\mathrm{m}_{e}}{\mathrm{e}} \int d^{3} r \mathbf{r} \times \mathbf{j}
$$

stands for the angular momentum of the SC current. The effective interaction (10), is mandated by symmetry and is, therefore, parameter free. Correspondingly, the mechanism of decoherence provided by this effect is universal.

Based upon Eq. (10) the rate of the transition from $|1\rangle$ to $|0\rangle$ is given by

$$
\Gamma_{s}=2 \hbar<0|\hat{\mathbf{L}}| 1>\left[J_{\text {env }}(\Delta)\right]<1|\hat{\mathbf{L}}| 0>,
$$

where $J_{e n v}(\Delta)$ is the spectral function of the environmental coupling for phonons,

$$
J_{e n v}(\Delta)=\pi \sum_{\mathbf{k}, i}\langle\mathbf{k}, i|\hat{\mathbf{\Omega}}| 0\rangle\langle 0|\hat{\mathbf{\Omega}}| \mathbf{k}, i\rangle \delta\left(\Delta-\hbar \omega_{\mathbf{k} i}\right)
$$

$\mathbf{k}, i$, and $\omega_{\mathbf{k} i}$ denote the wave vector, polarization, and the frequency of the phonon, respectively. Further computation along the lines of Ref. 11 yields

$$
\begin{aligned}
\Gamma_{s}(T) & =\frac{\hbar L^{2}}{12 \pi \rho} k_{s}^{5} \operatorname{coth}\left[\frac{\Delta}{2 k_{B} T}\right] \\
& =\frac{\mathrm{m}_{e}^{2}}{3 \pi \hbar \mathrm{e}^{2}} \frac{J^{2} a^{2}}{\rho} k_{s}^{5} \operatorname{coth}\left[\frac{\Delta}{2 k_{B} T}\right]
\end{aligned}
$$

where $\rho$ is the mass density of the solid matrix and $k_{s}=$ $2 \pi / \lambda_{s}$ is the wave number of the emitted sound.
It is important to notice that the decoherence rate of Eq. (14) is proportional to the fifth power of $f_{0}$ and to the fourth power of the size of the SQUID. It is inversely proportional to the fifth power of the speed of the transverse sound, making it important to use solid matrices (substrates) of high shear modulus. For practical values of the parameters: $k_{B} T \leq \Delta, R \sim 0.1 \mu \mathrm{m}, \quad J \sim 0.1 \mu \mathrm{A}$, $\rho \sim 5 \mathrm{~g} / \mathrm{cm}^{3}, \quad v_{s} \sim 5 \times 10^{3} \mathrm{~m} / \mathrm{s}$ and $f_{0} \sim 5 \times 10^{9} \mathrm{~s}^{-1}$ (that is, $\lambda_{s} \sim 1 \mu \mathrm{m}$ ), Eq. (14) gives $\Gamma_{s} \sim 1 \mathrm{~s}^{-1}$. Consequently, the above mechanism of decoherence should not be of great concern for a small SQUID. For a large SQUID the situation will be quite different, as is discussed below.

We should emphasize that Eq. (14) can be used for the estimate of the decoherence rate only at $R \leq \lambda_{s} \equiv v_{s} / f_{0}$. In experiments of Ref. 5 and Ref. 8 the size the SQUID was large in comparison with $\lambda_{s}$. The interaction of such a SQUID with phonons is non-local and cannot be treated by the above method. The non-local theory of the phonon emission by a large SQUID is developed in the next Subsection.

\section{B. Large SQUID}

For $R>\lambda_{s}$, one should employ the general form of the interaction given by Eq. (5). This term results in the coupled dynamics of the currents and the lattice displacements. However, in reality, the large difference between the ionic and electron masses makes the renormalization the SC dynamics insignificant. In what follows, we will ignore the effect of Eq. (5) on the spatial and temporal structure of the current and the SQUID flux $\Phi$ generated by the current. We shall be concerned with the fact that, due to Eq. (5), the currents serve as the source of phonons in the elastic equation. In other words, the solid lattice must take the recoil from the oscillating current. This effect is mandated by conservation laws and it leads to the decoherence of the quantum dynamics of the flux.

Inside a good conductor, either metal or a superconductor, the longitudinal electric fields are screened with a typical time scale of the plasma oscillations, $t \sim 10^{-15} \mathrm{~s}$. The flux dynamics is much slower. Consequently, the longitudinal phonons that change the local concentration of ions should be excluded from our consideration. This can be done by supplementing Eq. (5) with the condition (2). For the purpose of estimates we shall adopt the simplest model of uniform and isotropic elastic medium. Then, the energy of the free transverse phonon field is

$$
\mathcal{H}_{p h}=\int d^{3} r\left(\frac{1}{2} \rho \dot{\mathbf{u}}^{2}+\mu u_{i j}^{2}\right),
$$

where $\mu$ is the shear modulus of the solid and $u_{i j}=$ $\frac{1}{2}\left(\partial_{i} u_{j}+\partial_{j} u_{i}\right)$ is the strain tensor $\left(\sum_{i} u_{i i}\right.$ being zero for transverse phonons). In this model the torsional strains are described by just one elastic modulus $\mu=\rho v_{s}^{2}$. Accordingly, the transverse sound velocity $v_{s}$ is independent of the phonon polarization (which is orthogonal to 
the phonon wave vector). We shall further simplify our consideration by neglecting all differences in the actual material composition of the experimental setup, that is, by assuming that the phonon spectrum is the same inside and outside the part of the solid matrix that carries the $\mathrm{SC}$ current. This assumption, while not valid in experiment, should not significantly affect our estimate of the decoherence rate.

The canonical quantization of the phonon field yields:

$$
\begin{aligned}
\mathbf{u}(\mathbf{r}) & =\frac{1}{\sqrt{V}} \sum_{\mathbf{k}, i} \sqrt{\frac{\hbar}{2 \rho \omega_{\mathbf{k} i}}}\left(a_{\mathbf{k} i} \mathrm{e}^{i \mathbf{k r}}+a_{\mathbf{k} i}^{\dagger} \mathrm{e}^{-i \mathbf{k r}}\right) \mathbf{e}_{i} \\
\mathbf{\Pi}(\mathbf{r}) & =\frac{-i}{\sqrt{V}} \sum_{\mathbf{k}, i} \sqrt{\frac{\hbar \omega_{\mathbf{k} i} \rho}{2}}\left(a_{\mathbf{k} i} \mathrm{e}^{i \mathbf{k r}}-a_{\mathbf{k} i}^{\dagger} \mathrm{e}^{-i \mathbf{k r}}\right) \mathbf{e}_{i}
\end{aligned}
$$

Here $\boldsymbol{\Pi}=\rho \dot{\mathbf{u}}$ is the momentum of the phonons that is canonically conjugate to $\mathbf{u}, \omega_{\mathbf{k} i}=v_{s} k$ is the frequency of the phonon of the wave vector $\mathbf{k}$ and polarization $i$, and $V$ is the volume of the system. Due to the isotropy, $\omega_{\mathbf{k} i}$ for the transverse phonons $\left(\mathbf{k} \cdot \mathbf{e}_{i}=0\right)$ does not depend on the polarization. Substituting Eqs. (16) into Eq. (15) and Eq. (5) one obtains

$$
\begin{aligned}
\mathcal{H} & =\mathcal{H}_{p h}+\mathcal{H}_{i n t}=\sum_{\mathbf{k}, i} \hbar \omega_{\mathbf{k} i}\left(a_{\mathbf{k} i}^{\dagger} a_{\mathbf{k} i}+\frac{1}{2}\right) \\
& +\frac{i}{\sqrt{V}} \sum_{\mathbf{k}, i} \frac{\mathrm{m}_{e}}{\mathrm{e}}\left(\frac{\hbar \omega_{i \mathbf{k}}}{2 \rho}\right)^{1 / 2}\left(\mathbf{j}_{\mathbf{k}} \cdot \mathbf{e}_{i}\right)\left(a_{\mathbf{k} i}-a_{\mathbf{k} i}^{\dagger}\right)
\end{aligned}
$$

where $\mathbf{j}_{\mathbf{k}}=\int d^{3} r \mathbf{j} \exp (-i \mathbf{k r})$ is the spatial Fourier component of the current density $\mathbf{j}(\mathbf{r})$, and the summation is over $\mathbf{k}$ and $i$ satisfying $\mathbf{k} \cdot \mathbf{e}_{i}=0$. The Fermi golden rule, then, yields the following expression for the decoherence rate:

$$
\begin{aligned}
\Gamma_{p h}= & \frac{2 \pi}{\hbar} \frac{\mathrm{m}_{e}^{2}}{2 \mathrm{e}^{2} \rho} \Delta \operatorname{coth}\left[\frac{\Delta}{2 k_{B} T}\right] \times \\
& \sum_{i} \int \frac{d^{3} k}{(2 \pi)^{3}}\left|\left\langle 0\left|\left(\mathbf{j}_{\mathbf{k}} \cdot \mathbf{e}_{i}\right)\right| 1\right\rangle\right|^{2} \delta\left(\Delta-\hbar \omega_{i \mathbf{k}}\right),
\end{aligned}
$$

where $|0\rangle$ and $|1\rangle$ are given by Eqs. (7) and (8).

We shall now compute $\mathbf{j}_{\mathbf{k}}$. The SC current can be written in terms of the SC phase $\varphi$ and the vector potential A,

$$
\mathbf{j}=\frac{c}{4 \pi \lambda_{L}^{2}}\left(\frac{\Phi_{0}}{2 \pi} \nabla \varphi-\mathbf{A}\right)
$$

with $\Phi_{0}=h c / 2 \mathrm{e}$ and $\lambda_{L}$ being the flux quantum and the London penetration length, respectively. The vector potential satisfies the Maxwell equation,

$$
\nabla \times \nabla \times \mathbf{A}=\frac{4 \pi}{c} \mathbf{j},
$$

with the current density $\mathbf{j}$ given by Eq. (19) inside the SC loop and by $\mathbf{j}=0$ outside the loop.

To simplify calculations we shall adopt the ring geometry of the SQUID. In the presence of the flux $\Phi$, the phase $\varphi$ winds around the ring by $\varphi_{J}=2 \pi \Phi / \Phi_{0}$, which is the Josephson phase in the junction cutting the ring. We shall study the problem in cylindrical coordinates $(z, r, \phi)$, with the $Z$-axis passing through the center of the ring perpendicular to its plane, $r$ standing for the radial coordinate, and $\phi$ being the polar angle in the plane of the ring. The solution for the phase is

$$
\varphi=\frac{\varphi_{J} \phi}{2 \pi}=\frac{\Phi}{\Phi_{0}} \phi
$$

Due to the cylindrical symmetry of the currents, the only non-zero component of the vector potential is $A \equiv$ $A_{\phi}(r, z)$. Then, inside the ring, Eq. (20) reduces to

$$
-\frac{\partial}{\partial r}\left[\frac{1}{r} \frac{\partial}{\partial r}(r A)\right]-\frac{\partial^{2} A}{\partial^{2} z}+\lambda_{L}^{-2} A=\frac{\Phi}{2 \pi \lambda_{L}^{2}} \frac{1}{r},
$$

while outside the ring one has

$$
-\frac{\partial}{\partial r}\left[\frac{1}{r} \frac{\partial}{\partial r}(r A)\right]-\frac{\partial^{2} A}{\partial^{2} z}=0 .
$$

These equations must be accompanied by the boundary conditions for $A$ and for non-zero components of the magnetic field,

$$
H_{r}=-\frac{\partial A}{\partial z}, \quad H_{z}=\frac{1}{r} \frac{\partial(r A)}{\partial r} .
$$

In this paper we shall not pursue the exact solution of the problem for the finite cross-section of the ring carrying the current. Instead, we will make use of a thin-ring approximation in which the thickness of the ring $\sqrt{b}$, where $b$ stands for the area of the wire crossection, is small compared to its radius $R$ as well as to $\lambda_{L}$. Then, in cylindrical coordinates, the only non-zero component of the current density is $j_{\phi}(z, r)$. It equals $J / b$ inside the ring and zero outside the ring. At $k \sqrt{b} \ll 1$ the Fourier transform of such a distribution of the current is

$$
\mathbf{j}_{\mathbf{k}}=-i 2 \pi R J_{1}\left(k_{\perp} R\right) J \mathbf{n}_{\mathbf{k}}
$$

where $\mathbf{n}_{\mathbf{k}} \perp \mathbf{k}$ is the unit vector in the plane of the ring, $k_{\perp}=k \sin \theta, \theta$ is the angle between $\mathbf{k}$ and the $Z$-axis, and $J_{1}\left(k_{\perp} R\right)$ stands for the Bessel function of the first order.

The quantization procedure consists of assigning the operator $\hat{J}$ to the total current $J$. In the two-level approximation, one introduces the states $| \pm\rangle$ of the current operator such that $\pm J$ are the respective eigenvalues: $\hat{J}| \pm\rangle= \pm J| \pm\rangle$. In terms of the angular momentum operator, these states are identical to those in Eq. (6), that is $|+\rangle \equiv|\uparrow\rangle$ and $|-\rangle \equiv|\downarrow\rangle$. Tunneling between these two degenerate states produces new states, Eq. (7) and Eq. (8), which are split by the energy $\Delta$. These 
states are characterized by the zero current, $\langle 0|\hat{J}| 0\rangle=0$ and $\langle 1|\hat{J}| 1\rangle=0$. The transition matrix element is $\langle 0|\hat{J}| 1\rangle=J$. Substituting Eq. (25) into Eq. (18) we get

$$
\begin{gathered}
\Gamma_{p h}=\frac{2 \pi \mathrm{m}_{e}^{2}}{\hbar \mathrm{e}^{2}} \frac{J^{2} R^{2}}{\rho} \operatorname{coth}\left[\frac{\Delta}{2 k_{B} T}\right] \times \\
k_{s}^{3} \int_{0}^{1} d \cos \theta J_{1}^{2}\left(k_{s} R \sin \theta\right) .
\end{gathered}
$$

The limit of $k_{s} R \ll 1$ corresponds to a small SQUID. It is easy to see that in this limit $\Gamma_{p h}$ is proportional to $k_{s}^{5}$ and Eq. (26) becomes Eq. (14). In the limit of a large SQUID, $k_{s} R \gg 1$, we find

$$
\Gamma_{p h}=\frac{\pi \mathrm{m}_{e}^{2}}{\hbar \mathrm{e}^{2}} \frac{J^{2} R}{\rho} k_{s}^{2} \operatorname{coth}\left[\frac{\Delta}{2 k_{B} T}\right] .
$$

Based upon Eq. (27), let us make an estimate of the decoherence rate for, e.g., the experiment of Ref. 5 . At $k_{B} T \leq \Delta$, for $R \sim 0.1 \mathrm{~mm}, \quad J \sim 3 \mu \mathrm{A}, \quad \rho \sim 8 \mathrm{~g} / \mathrm{cm}^{3}$, $v_{s} \sim 5 \times 10^{3} \mathrm{~m} / \mathrm{s}$ and $f_{0} \sim 2 \times 10^{9} \mathrm{~s}^{-1}$ (that is, $\lambda_{s} \sim 2.5 \mu \mathrm{m}$ ), Eq. (27) gives $\Gamma_{p h} \sim 10^{6} \mathrm{~s}^{-1}$. This is a significant decoherence rate that would limit the quality factor of the corresponding qubit by the value of about one thousand.

\section{Global noise}

Here we will compare the effect of the global noise uncontrolled rotations of the solid matrix as a whole at some angular velocity $\Omega_{G}(t)$ - with the above estimates for the local effects due to phonons. The $Z$-component of the global rotation removes the degeneracy between clockwise and counterclockwise current states. This is a particular case of the Barnett effect: A rotating solid develops magnetization proportional to the angular velocity of the rotation 12. In application to the SQUID this effect can be described by a two-state Hamiltonian written in the rotating coordinate frame:

$$
\mathcal{H}_{G}=-\Delta s_{x}-2 \hbar L \Omega_{G}(t) s_{z},
$$

where $s_{x, z}$ are spin- $1 / 2$ operators. We want to estimate the effect of the second term in Eq. (28) on coherent oscillations of the SC current. (Notice that for externally imposed rotations, the sign of this term is opposite to the sign of Eq. (10) that was written for phonons dynamically produced by SQUID oscillations in the laboratory frame.) For a macroscopic solid matrix, the characteristic correlation time of $\left\langle\Omega_{G}(t) \Omega_{G}(0)\right\rangle$ cannot be less than the time it takes the sound to travel across the matrix. Consequently, on the time scale $t \sim 1 / f_{0}$, random rotations of the equipment as a whole are slow enough to permit the treatment of Eq. (28) within the adiabatic approximation.

In the adiabatic approximation the eigenvalues and eigenfunctions of the Hamiltonian (28) are

$$
\begin{aligned}
\epsilon_{ \pm} & = \pm \sqrt{\Delta^{2}+\left[2 \hbar L \Omega_{G}\right]^{2}} \\
\psi_{ \pm} & =\frac{1}{\sqrt{2}}\left[C_{\mp}(t)|\uparrow\rangle \pm C_{ \pm}|\downarrow\rangle\right],
\end{aligned}
$$

where $C_{ \pm}$are given by

$$
C_{ \pm}=\sqrt{1 \pm \frac{2 \hbar L \Omega_{G}}{\sqrt{\Delta^{2}+\left[2 \hbar L \Omega_{G}\right]^{2}}}} .
$$

One can see that random rotations of the system as a whole do not significantly perturb the states $|0\rangle$ and $|1\rangle$ given by Eq. (7) and Eq. (8) only if $\Omega_{G}$ satisfies

$$
\Omega_{G} \ll \frac{\omega_{0}}{2 L} .
$$

For a large SQUID with $L \sim 10^{10}$ and $\omega_{0} \sim 10^{10} \mathrm{~s}^{-1}$, this gives $\Omega_{G} \ll 1 \mathrm{~s}^{-1}$, which must be of practical importance in the situation when the equipment is subjected to random movements. For a small SQUID, with small $L$ and practical values of $\omega_{0}$, the condition (31) can be satisfied by a very large margin.

The time dependence of $\Omega_{G}$ is another factor. The adiabaticity implies that

$$
\mathcal{A} \equiv \frac{\pi \omega_{0}^{2}}{4 L\left|\dot{\Omega}_{G}\right|} \gg 1
$$

Then, the main effect of the time dependence of the global rotations is generation of additional harmonics in the Rabi oscillations between clockwise and counterclockwise SC currents due to the time dependence of $\epsilon_{ \pm}$. The corresponding decoherence rate, $\Gamma_{G}$, can be estimated from the variation of $\epsilon_{ \pm}$during one cycle, $2 \pi / \omega_{0}$, of the undisturbed Rabi oscillations. The global random rotations occur when the equipment is subjected to a random external torque. Let this torque result in an angular acceleration $\alpha=\dot{\Omega}_{G}$, so that the change of $\Omega_{G}$ during one cycle is $\delta \Omega_{G} \sim \alpha / \omega_{0}$. This gives

$$
\Gamma_{G} \sim \delta \epsilon_{+} / \hbar \sim \omega_{0} / \mathcal{A}
$$

where $\mathcal{A}=\pi \omega_{0}^{2} / 4 L|\alpha|$. Thus, the quality factor for the above mechanism is $Q=\mathcal{A}$. It is entirely determined by the adiabaticity of global rotations.

If the typical frequency of the mechanical noise is $\omega_{n}$, then $\alpha \sim \omega_{n} \Omega_{G}$. Assuming that the condition (31) is satisfied, this implies

$$
Q=\mathcal{A} \gg \omega_{0} / \omega_{n}
$$

In principle, at small $\omega_{0}$, the effect of the mechanical noise can overpower the decoherence from local phonon effects, which decrease as some power of $\omega_{0}$. However, for any practical values of $\omega_{0}$ and $\alpha$ in the coherence experiments, the adiabadicity factor $\mathcal{A}$ is very large, so that the decohering effect of the external global noise can be safely ignored.

We also would like to make the following interesting observation. Consider rotations $\Omega_{G}=\alpha t$ that last long 
enough to violate the condition (31). According to Eq. (30), as time goes from $-\infty$ to $+\infty$, the states $\psi_{ \pm}$of Eq. (29) switch between $|\uparrow\rangle$ and $|\downarrow\rangle$. This, however, is true only in the limit of $|\alpha| \rightarrow 0$. At finite $|\alpha|$ the answer depends on the adiabaticity factor $\mathcal{A}$. If at $t=-\infty$ the SQUID is prepared in, e.g., the state with the clockwise SC current, then, the probability for the SQUID to switch to the counterclockwise current at $t=+\infty$ is given by the Landau-Zener formula 13

$$
P_{L Z}=1-e^{-\mathcal{A}} \text {. }
$$

This probability is high if the angular acceleration satisfies $\alpha \leq \alpha_{c}=\pi \omega_{0}^{2} / 4 L$. Consequently, at low tunneling rate the relatively slow mechanical rotation can provide the quantum-mechanical switching between clockwise and counterclockwise currents. It should be noted, however, that the Landau-Zener transitions can also be generated by the magnetic field. Thus, the above effects of uniform rotations can only be observed if the SQUID is shielded from the magnetic fields with an accuracy $\mu_{B} H<\hbar \Omega_{G}$.

\section{DECOHERENCE DUE TO PHOTONS}

The problem of decoherence due to the emission of photons of frequency $f_{0}$ is very similar to the problem of the emission of phonons. The main difference is that the vacuum wavelength of the light, $\lambda_{l}=c / f_{0}$, is typically large compared to the size of the SQUID that exhibits quantum oscillations of the current. The electromagnetic radiation by such a SQUID into the open space is equivalent to the radiation of a point magnetic dipole. If, however, the SQUID is shielded by a metal placed at a distance that is comparable to or smaller than $\lambda_{l}$, the decoherence rate becomes strongly geometry-dependent. These two problems are considered in the following two Subsections.

\section{A. Decoherence in the open space}

If the wavelength of the emitted photons is large compared with the SQUID size, then the SQUID can be treated as a point particle with an angular momentum $\mathbf{L}$ which is perpendicular to the SQUID loop. This angular momentum interacts with the photon field via Zeeman Hamiltonian

$$
\mathcal{H}_{Z}=-\mu_{B} L_{z} H_{z}
$$

where $H_{z}$ is the $Z$-component of the magnetic field of the vacuum photons. We are interested in the transition between the tunnel-splitted quantum states given by Eq. (7) and Eq. (8). The expression for the rate is similar to Eq. (12), where the spectral density of the photons can be obtained by either quantizing $\mathbf{H}(\mathbf{r})$ or taking $\left\langle\left|H_{\omega}\right|^{2}\right\rangle$ from the theory of electromagnetic fluctuations 14.15.
The Fermi golden rule then yields the following expression for the decoherence rate

$$
\begin{aligned}
\Gamma_{Z}(T) & =\frac{4}{3 \hbar} \mu_{B}^{2} L^{2} k_{l}^{3} \operatorname{coth}\left[\frac{\Delta}{2 k_{B} T}\right] \\
& =\frac{4}{3 \hbar c^{2}} J^{2} a^{2} k_{l}^{3} \operatorname{coth}\left[\frac{\Delta}{2 k_{B} T}\right],
\end{aligned}
$$

where $k_{l}=\Delta / \hbar c$ is the wave vector of the emitted light. It is proportional to the third power of $f_{0}$ as compared to the fifth power of $f_{0}$ in Eq. (14). The reason for the difference is the additional time derivative of the boson field in the Hamiltonian of Eq. (10) as compared to Eq. (36).

Notice that the identical result for $\Gamma_{Z}$ follows from

$$
\Gamma_{Z}(0)=\frac{I}{\hbar \omega_{0}},
$$

where 15

$$
I=\frac{4}{3 c^{3}}|\ddot{\mathbf{M}}|^{2}
$$

is the intensity of the magnetic dipole radiation due to the classical dynamics of the magnetic moment $\mathbf{M}=\mu_{B} \mathbf{L}$.

For a small SQUID (as defined above in Sec. II) with $R \sim \lambda_{s} \sim 100 \mathrm{~nm}$ and $J \sim 0.1 \mu \mathrm{A}$, Eq. (37), at $f_{0} \sim 10^{10} \mathrm{~s}^{-1}$ and $k_{B} T \leq \Delta$, gives negligible decoherence, $\Gamma_{Z} \sim 10^{-8} \mathrm{~s}^{-1}$. However, for a large SQUID with $R \sim 100 \mu \mathrm{m}$ and $J \sim 3 \mu \mathrm{A}$, at $f_{0} \sim 10^{10} \mathrm{~s}^{-1}$ and $k_{B} T \ll$ $\Delta$, one obtains $\Gamma_{Z} \sim 10^{7} \mathrm{~s}^{-1}$. Thus, for a large SQUID, the radiation of photons into the open space can easily reduce the quality factor of the SQUID down to one hundred.

The decoherence rate can be decreased by choosing a double-loop geometry with equal areas of the single loops and equal currents flowing in the opposite directions, as was actually done in Ref. 5. In that case, the total magnetic moment of the system is zero and the radiation is of the quadrupolar nature. If the magnetic moment is compensated exactly (which must be difficult to achieve in experiment) the radiation rate will be reduced by a factor $(k R)^{2} \ll 1$. At a finite compensation, $\gamma=\Delta M / M<1$, the decoherence rate (37) acquires a factor $\gamma^{2}$.

Here we have neglected the effects of dc and lowfrequency adiabatic ac magnetic fields $H(t)$. These effects are equivalent to the effects of global rotations studied in the Subsection II-C. To estimate them quantitatively one should replace $\Omega_{G}$ and $\alpha$ by $\omega_{H}=\mathrm{e} H / 2 m_{e} c$ and $\omega_{a c} \omega_{H}$ respectively, where $\omega_{a c}$ is the typical frequency of the ac field. Then, conditions (31) and (32) become

$$
\omega_{H} \ll \frac{\omega_{0}}{2 L}
$$

and

$$
\mathcal{A} \equiv \frac{\pi \omega_{0}^{2}}{4 L \omega_{a c} \omega_{H}} \gg 1,
$$


respectively. In a two-loop design, where the magnetic moment is compensated by a factor $\gamma=\Delta M / M<1$, $L$ in Eqs. (40) and (41) should be replaced by $\gamma L$.

For, e.g, a small SQUID with $L \sim 100$ and no compensation, Eq. (40) at $\omega_{0} \sim 10^{10} \mathrm{~s}^{-1}$ translates into $H \ll$ 10 Oe. For a large SQUID with $\gamma=10^{-2}, L=10^{10}$ and $\omega_{0} \sim 10^{10} \mathrm{~s}^{-1}$, the fields that do not disturb the states $|0\rangle$ and $|1\rangle$ should satisfy $H \ll 10^{-5}$ Oe. If Eq. (40) is satisfied, then substituting it into Eq. (41) one obtains the following relation for the quality factor coming from the low-frequency magnetic noise alone:

$$
Q=\mathcal{A} \gg \omega_{0} / \omega_{a c}
$$

\section{B. Decoherence in the presence of metal shielding}

We shall now study the case when the SQUID loop is adjacent to a metal sheet parallel to the plane of the loop. Following published experiments, we shall assume that the distance between the SQUID and the shielding, $d$, is much smaller than the wavelength of the vacuum electromagnetic radiation $\lambda_{l}$. In that case the radiation becomes strongly renormalized by the conducting medium and the formulas of the previous Subsection can no longer be used. Now the main source of decoherence is the dissipative current in the metal shielding induced by the ac fields of the SQUID. Correspondingly, the decoherence rate can be computed as

$$
\Gamma_{M}=\frac{P}{\hbar \omega_{0}}
$$

where $P$ is the power absorbed by the shielding. Let $\mathbf{H}=\mathbf{H}_{\omega}(\mathbf{r}) \exp \left(i \omega_{0} t\right)$ be the magnetic field generated by the oscillating current in the SQUID. Then $P$ is given by 15

$$
P=\frac{c}{16 \pi} \sqrt{\frac{\omega_{0}}{2 \pi \sigma}} \oint\left\langle\left|\mathbf{H}_{\omega}\right|^{2}\right\rangle d f
$$

where $\sigma$ is the electric conductivity of the shielding, $\langle\ldots\rangle$ means quantum-mechanical average, and the integration goes over the metal surface facing the SQUID.

Eq. (44) can be used when the thickness of the skin layer, $\delta=c / \sqrt{2 \pi \sigma \omega_{0}}$, is small compared to the thickness of the shielding metal, $D$, but large compared to the mean free path of electrons of the metal, $l_{0}$. These conditions were apparently fulfilled in the experiment of Ref. 5 for which we estimate $\delta \sim 1 \mu \mathrm{m}$ and $l_{0}<0.1 \mu \mathrm{m}$ at $D \sim 8 \mu \mathrm{m}$. The condition $d \ll \lambda_{\text {l }}$ allows one to use the quasistationary approximation 15 to obtain $\mathbf{H}$. In this approximation the field is formed by two current loops, one being the mirror image of the other with respect to the surface of the shielding. At the metal surface, $z=d$, this field has the tangential component only, $H_{r}(r)$. For a thin circular loop carrying the electric current $J(t)=J \exp \left(i \omega_{0} t\right)$, it is given by

$$
H_{r}=\frac{4 J R d}{c} \int_{0}^{\pi} \frac{d \phi \cos \phi}{\left(d^{2}+r^{2}+R^{2}-2 r R \cos \phi\right)^{3 / 2}},
$$

which can be expressed in terms of the elliptic integrals 15.

Substituting Eq. (45) into Eq. (44), one obtains

$$
\Gamma_{M}=\frac{2}{h c} \frac{J^{2}}{\sqrt{\sigma f_{0}}} F(R / d)
$$

where the function $F$ is given by

$F(\xi)=\xi^{2} \int_{0}^{\infty} d x x\left[\int_{0}^{\pi} \frac{\cos \phi d \phi}{\left(1+x^{2}+\xi^{2}-2 x \xi \cos \phi\right)^{3 / 2}}\right]^{2}$.

In the two limiting cases of small and large $d$, the geometrical factor 47 ) reduces to

$$
\begin{aligned}
& F=\frac{l}{4 d}, \quad d \ll R \\
& F=\frac{3 a^{2}}{32 d^{4}}, \quad d \gg R .
\end{aligned}
$$

Here we have introduced the length and the area of the current loop, $l=2 \pi R$ and $a=\pi R^{2}$, respectively, in order to emphasize the fact that the above limiting expressions are correct for flat current loops of arbitrary shape. Numerical analysis shows that these expressions hold for $d / R \leq 0.3$ and $d / R>5$, correspondingly. For the double-loop with a compensated magnetic moment due to equal single-loop currents flowing in the opposite directions, the decoherence rate practically does not change in the limit of $d \ll R$. In the opposite limit of $d \gg R$, it reduces by a factor $(R / d)^{2}$.

Note that the frequency dependence of the rate (46) follows from the frequency dependence of the skin depth, $\delta \propto 1 / \sqrt{f_{0}}$. Then the Maxwell equation gives $E \propto$ $\delta \cdot(\partial H / \partial t) \propto \sqrt{f_{0}}$ for the electric field in the skin layer and the dissipation rate due to Joule's power losses, $\sigma E^{2} \delta$, becomes proportional to $\sqrt{f_{0}}$. Divided by $f_{0}$ in Eq. (43), it gives the $1 / \sqrt{f_{0}}$ dependence of the decoherence rate. If the thickness of the metal shielding $D$ is smaller than $\delta$, the electric and magnetic fields are not significantly modified by the shielding, so that $E \propto f_{0} H$, with the proportionality factor determined by the geometry of the SQUID and its distance to the shielding. Thus, in the low-frequency limit $(D<\delta)$, the dissipation in the shielding $P \sim \sigma E^{2} D$ is proportional to $f_{0}^{2}$ and $\Gamma_{M}$ due to shielding is proportional to $f_{0}$.

For $J \sim 3 \mu \mathrm{A}, \sigma=3 \times 10^{17}$ e.m.u., $f_{0} \sim 2 \times 10^{9} \mathrm{~s}^{-1}$, and $R \sim d$, Eq. (46) gives $\Gamma_{M} \sim 10^{9} \mathrm{~s}^{-1}$, which was, probably, the case in the experiment of Ref. 5. This shows that for a SQUID carrying a microampere current the above mechanism can provide a very high decoherence rate. Notice that $\Gamma_{M}$ can be drastically reduced by increasing the distance, $d$, between the SQUID and the shielding. Indeed, according to Eqs. (48), $\Gamma_{M} \propto d^{-4}$ at $R \ll d \ll \lambda_{l}$. Too large $d$, however, would reduce the effectiveness of the 
shielding in protecting the SQUID from external radio signals. Choosing smaller SQUIDS operating at smaller currents should be more beneficial for qubit designs.

\section{CONCLUSIONS}

We have studied generic mechanisms of decoherence mandated by the conservation laws - emission of phonons and photons of the oscillation frequency $f_{0}$. Our practical conclusions are as follows:

The decoherence due to the above mechanisms scales as the second power of the current.

For small SQUIDS of size $R<v_{s} / f_{0}$, the decoherence due to the emission of phonons at $T \leq \Delta$ is negligible.

For large SQUIDs of size $R \gg v_{s} / f_{0}$, the emission of phonons can significantly limit the quality factor. The corresponding decoherence rate scales linearly with the size of the SQUID and quadratically on the oscillation frequency.

In the absence of the metal shielding, the emission of photons is negligible for small SQUIDs but becomes significant for large SQUIDs. It scales as the fourth power of the size of the SQUID and as the third power of the oscillation frequency.

Decoherence due to the shielding strongly depends on the geometry of the experimental setup. It may completely destroy the coherence in large SQUIDs and can be the main mechanism of decoherence in small SQUIDs. The shielding must be provided by a metal sheet of thickness greater than the skin layer at the oscillation frequency $f_{0}$. To achieve small decoherence, the distance to the shielding, while small in comparison with $c / f_{0}$, should be considerably greater than the loop size.

The effects of the external mechanical and magnetic noise are proportional to the total magnetic moment of the SQUID, making small SQUIDS less susceptible to the noise than large SQUIDs.

\section{ACKNOWLEDGMENTS}

We thank J. Friedman and B. Svistunov for useful discussions. This work has been supported by the DOE Grant No. DE-FG-2-93ER45487.

${ }^{1}$ A. O. Caldeira and A. J. Leggett, Ann. Phys. (N.Y.) 149, 374 (1983).

2 J. M. Martinis, M. H. Devoret, and J. Clarke, Phys. Rev. Lett. 55, 1543 (1985).
${ }^{3}$ J. Clarke, A. N. Cleland, M. H. Devoret, D. Esteve, and J. M. Martinis, Science 239, 992 (1988).

${ }^{4}$ A. J. Leggett, S. Chakravarty, A. T. Dorsey, M. P. Fisher, A. Garg, W. Zwerger, Rev. Mod. Phys. bf 59, 1 (1987).

${ }^{5}$ J. R. Friedman, V. Patel, W. Chen, S. K. Tolpygo, and J. E. Lukens, Nature 406, 43 (2000).

${ }^{6}$ C. H. van der Wal, A. C. J. ter Haar, F. K. Wilhelm, R. N. Schouten, C. J. P. M. Harmans, T. P. Orlando, S. Lloyd, and J. E. Mooij, Science 290, 773 (2000).

${ }^{7}$ D. Vion, A. Aassime, A. Cottet, P. Joyez, H. Pothier, C. Urbina, D. Esteve, M. Devoret, Science 296, 886 (2002).

${ }^{8}$ Y. Yu, S. Han, X. Chu, S. Chu, Z. Wang, Science 296, 889 (2002).

${ }^{9}$ See, e.g., A. J. Leggett, Science 296, 861 (2002).

${ }^{10}$ E. M. Chudnovsky, Phys. Rev. Lett. 72, 3433 (1994).

${ }^{11}$ E. M. Chudnovsky and X. Martinez-Hidalgo, Phys. Rev. B66, 054412 (2002).

12 S. J. Barnett, Rev. Mod. Phys. 7, 129 (1937).

${ }^{13}$ L. D. Landau, Phys. Z. Sowjetunion 2, 46 (1932); C. Zener, Proc. R. Soc. London, Ser. A, bf 137, 696 (1932).

${ }^{14}$ E. M. Chudnovsky and D. A. Garanin, Phys. Rev. Lett. 89, 157201 (2002).

${ }^{15}$ L. D. Landau and E. M. Lifshitz, Electrodynamics of Continuous Media (Nauka,Moscow, 1982). 